\title{
Wave Erosion of Cohesive and non-Cohesive Embankments: Laboratory Experiments
}

\author{
Yavuz Ozeren ${ }^{1, *}$, and Daniel Wren ${ }^{2}$ \\ ${ }^{1}$ National Center for Computational Hydroscience and Engineering, The University of Mississippi, \\ 344 Brevard Hall, University, MS, 38677, USA \\ ${ }^{2}$ United States Department of Agriculture, Agricultural Research Service, National Sedimentation \\ Laboratory, 598 McElroy Drive, Oxford, MS, 38655, USA
}

\begin{abstract}
Laboratory experiments were carried out in a wave tank to investigate wave induced beach profile evolution and scarp recession on cohesive and non-cohesive model embankments. The cohesive embankment was constructed using a natural soil mixture, and the noncohesive embankment was constructed using cylindrical shaped polyethylene terephthalate (PET) pellets of uniform size. A standardized packing procedure was used for both cohesive and non-cohesive embankments. The onshore face slope of the embankment was approximately 30 degrees. The morphological evolution of the embankment was monitored using water level sensors and a time-lapse camera. Waves quickly eroded the bank face near the free surface and formed a scarp. While an equilibrium profile was established relatively quickly and at a much steeper slope for the non-cohesive embankment, the cohesive embankment continued to erode for a much longer period. The scarp of the cohesive embankment retreated by a series of wave undercutting and slump failures. An empirical relationship between incident wave height and embankment erosion was established for the tested conditions. The measured beach profiles were compared with the developed analytical solution.
\end{abstract}

\section{Introduction}

Irrigation in the lower Mississippi River basin relies heavily on groundwater resources. The Mississippi River Valley alluvial aquifer has been significantly depleted in the last two decades due to withdrawals of water for irrigation. A number of measures to ensure sustainable irrigation water supply in the region are being used. One of the solutions to the problem is the use of on-farm reservoirs for irrigation water storage. The embankments of these reservoirs are often constructed using local soils that are low in clay content, and the inner slopes of the embankments are subjected to continuous erosion and retreat due to wind generated waves. A natural beach slope is in local equilibrium when the erosion and deposition of the beach material is in equilibrium. The inner slopes of the irrigation reservoir embankments are much steeper than the natural beach slope for typical wave sizes

*Corresponding author: yozeren@,ncche.olemiss.edu 
observed in these reservoirs. Strong winds, combined with high water levels, can quickly initiate an erosion and retreat process that eventually establishes an equilibrium profile. Wave breaking and runup erodes the beach landward, undercutting an existing scarp or forming a new scarp, which provides sediments to the developing beach profile. Some of the sediments are carried in the offshore and longshore direction by wave-induced or other types of currents.

The processes and their consequences were observed in numerous irrigation reservoirs in Arkansas, USA. [1] showed that almost $80 \%$ of the embankments of 148 surveyed reservoirs had block failures with near-vertical slopes. Figure $1 \mathrm{~b}$ compares an as-built cross-section with two surveys from 2015 and 2017 for a typical embankment of an irrigation reservoir at the University of Arkansas at Pine Bluff Lonoke Demonstration Farm. For this particular case, up to 4-m horizontal embankment loss was observed in 10 years. [2] measured $3 \mathrm{~m}$ of horizontal embankment loss in another irrigation reservoir in less than 6 months.

Little research has been done on the wave erosion of cohesive embankments. The available work is typically concentrated on the erosion of sandy beach dunes by storm waves ([3], [4] and [5]). The main focus of this study is to experimentally investigate the wave induced beach profile evolution and scarp recession on cohesive and non-cohesive model embankments. This paper presents the results of the laboratory experiments.

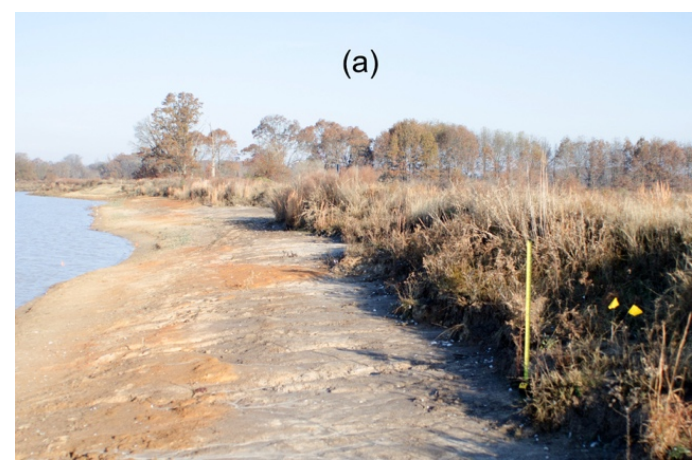

(b)

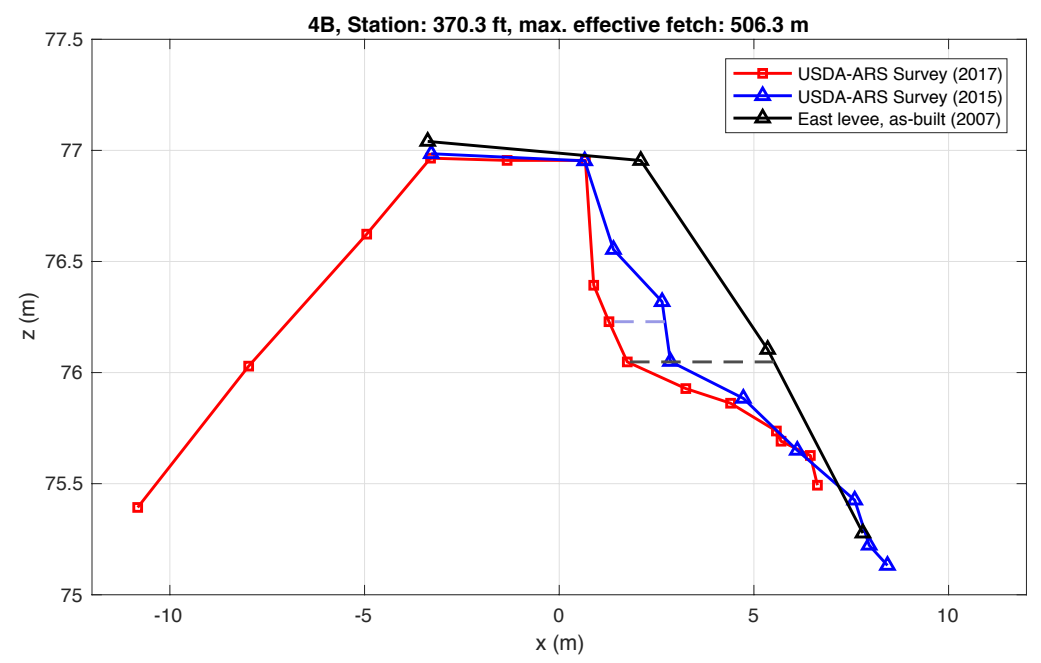

Fig. 1. (a) Typical embankment inner slope, (b) comparison of the cross-sections in 2015 and 2017 with as build cross-sections. 


\section{Experimental Setup}

\subsection{Wave tank}

Experiments were carried out in a $20.6 \mathrm{~m}$ long, $0.7 \mathrm{~m}$ wide, and $1.2 \mathrm{~m}$ deep laboratory wave tank at the USDA-ARS, National Sedimentation Laboratory in Oxford, MS (Fig. 1). The wave tank was equipped with a computer-controlled piston type wave maker. The bottom of the wave tank was elevated $0.3 \mathrm{~m}$ by a false floor.

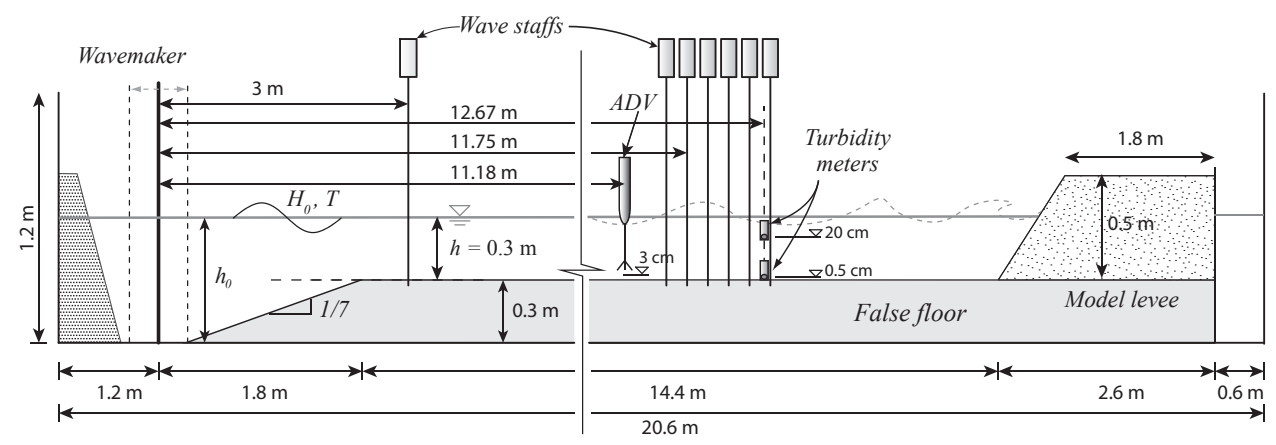

Fig. 2. Definition sketch of the wave tank setup.

\subsection{Embankment model}

The cohesive embankment was constructed using a natural soil mixture composed of $1 \%$ sand, 90\% silt and 9\% clay. A standardized packing procedure was used to construct each cohesive embankment. The soil was dried for 24 hours in an oven and crushed in a hammer mill to remove clumps. The soil was then mixed with water in a concrete mixer to achieve $10 \%$ moisture content. The dried soil was placed in the wave tank in $3 \mathrm{~cm}$ thick horizontal layers and packed using a vibrating plate and a heavy roller. The procedure was repeated until the height of the embankment was $50 \mathrm{~cm}$. The face of the embankment had a slope of approximately 30 degrees.

The non-cohesive embankment was constructed using cylindrical polyethylene terephthalate (PET) pellets of uniform size with a median diameter $\left(\mathrm{d}_{50}\right)$ of $2.87 \mathrm{~mm}$, density of $1.42 \mathrm{~kg} / \mathrm{m}^{3}$, and porosity of 0.34 (Fig. 3). The PET grains were packed using a concrete vibrator and leveled before each experiment. The face of the non-cohesive embankment also had a 30-degrees slope.

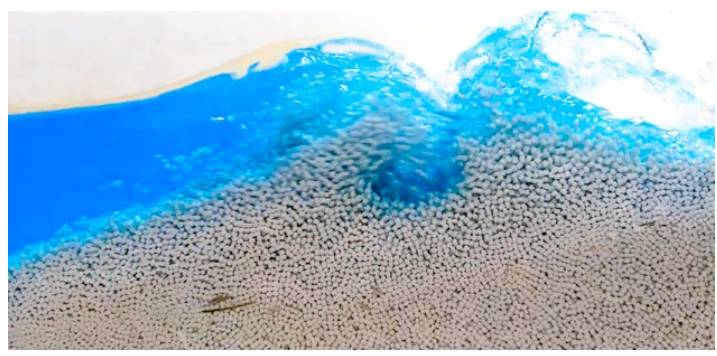

Fig. 3. Wave breaking on model beach with artificial non-cohesive material. 


\subsection{Instrumentation}

Wave height was measured by seven capacitance type water level sensors with a $30 \mathrm{~Hz}$ sampling rate. One of the sensors was located just onshore of the wave paddle to measure the incident wave height, and the remaining sensors were distributed offshore of the model embankment to measure the incident and reflected wave heights. The morphological evolution of the embankment was monitored using a time-lapse digital camera with a pixel resolution of 3696 by 2448 . The camera was placed $6 \mathrm{~m}$ away from the wave tank wall, which provided $0.3 \mathrm{~mm}$ per pixel resolution. The time lapse duration was $12 \mathrm{~s}$ during the early stages and progressively increased up to 1 min intervals.

\section{Results and Discussion}

\subsection{Experiments}

After packing the embankment model for each test case, the water level in the wave tank was gradually raised up to the levee height and left at least one day to completely saturate the soil. Then, it was reduced to $30 \mathrm{~cm}$ just before the experiments begun. Regular waves of wave period $1.2 \mathrm{~s}$ and water depth $30 \mathrm{~cm}$ at varying heights were generated for all of the tested conditions (Table 1). During the experiments waves were generated for 10-min-long intervals followed by 3-min-long resting periods. In this paper, only experiments 070716 and 110117 will be compared.

Table 1. Experimental conditions.

\begin{tabular}{|c|c|c|}
\hline Experiment & $\mathbf{H}_{\mathbf{0}}(\mathbf{c m})$ & Material \\
\hline 062915 & 4.0 & Natural soil \\
\hline 070716 & 4.2 & Natural soil \\
\hline 072915 & 5.4 & Natural soil \\
\hline 121415 & 5.5 & Natural soil \\
\hline 060815 & 6.5 & Natural soil \\
\hline 081715 & 8.2 & Natural soil \\
\hline 110107 & 4.1 & PET pellets \\
\hline 112017 & 7.2 & PET pellets \\
\hline
\end{tabular}

\subsection{Data Analysis}

Water surface elevation was monitored at fixed locations along the wave tank by seven wave staffs. When the incident wave interacted with the model embankment, some of the waves were reflected, which created partial standing waves. Therefore, the measured signal at each gauge provided the water surface displacement at some location along the partial standing wave. Using linear wave theory, the recorded, reflected and incident wave heights were separated. The wave heights listed in Table 1 were obtained through this procedure. 
At the beginning, reflected wave height was $15 \%-10 \%$ of the incident wave height. Within the first 10-min interval, as the embankment face eroded and the beach slope got milder, the reflected wave height reduced below $10 \%$ of the incident wave height. In general, the reflected wave height was higher for the experiment with non-cohesive embankment material.

The embankment profiles were digitized from the time-lapse images using a custom developed MATLAB tool. The experiments with PET pellets had a well-defined interface between the embankment surface and the dyed-blue water, which enabled the use of an automated image analysis procedure to identify the embankment erosion profiles. High suspended sediment concentration for natural soil experiments necessitated manually digitized profiles.

Figure 4 shows an envelope of digitized profiles for the cohesive and non-cohesive embankments. The colors of the profiles transition from purple to green to indicate forward movement in time. Dashed lines are the profiles within the first 10-minute-long run. Horizontal axis scales are the same in both plots. In both experiments, a scarp was quickly formed before receding onshore as waves continued to erode the embankment face. The non-cohesive embankment reached the equilibrium profile much more quickly, such that the profile did not change significantly after the first 10-minute interval. It also had a much steeper final profile than the cohesive embankment. The scarp retreat for the cohesive embankment occurred in discrete slump failures that can be seen in Fig. 4.
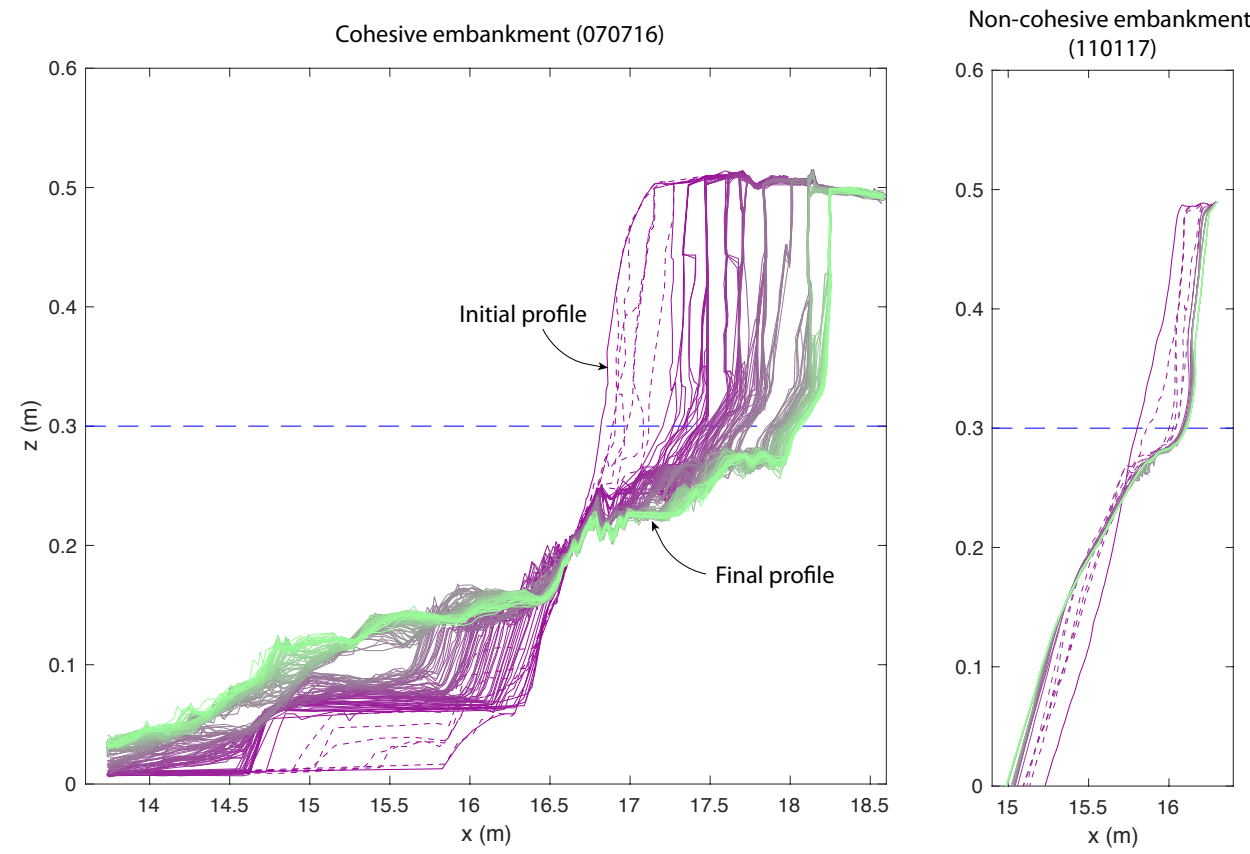

Fig. 4. Comparison of the eroded profiles of the cohesive (070716) and non-cohesive (110117) embankments.

As shown in Fig. 5a, the cohesive and non-cohesive embankment shapes after 30 minutes of wave action were similar. The scarp toe was approximately at the still water level in both pictures, and the toes of the embankments were at the same distance from the scarp location. The geometry of the non-cohesive embankment did not change significantly after the profile shown in Fig. 5a, while the cohesive embankment continued evolving at a reducing rate until the end of the experiment. The cohesive embankment after 33 hours of 
wave action is shown in Fig $5 b$. There are fundamental differences in the erosion process between sandy and cohesive shores ([4], [5]). The cohesive embankment is composed erodible materials with high silt content. The fine material of the cohesive embankment is eroded, and transported away from the nearshore zone. Unlike the sandy shore, the fine eroded material is permanently lost and cannot be recovered for a new equilibrium state.

Each embankment profile was compared with the initial embankment profile to calculate the eroded and deposited areas shown in Fig. 6. The intersection point P in Fig. 6 was essentially stationary for all experiments.

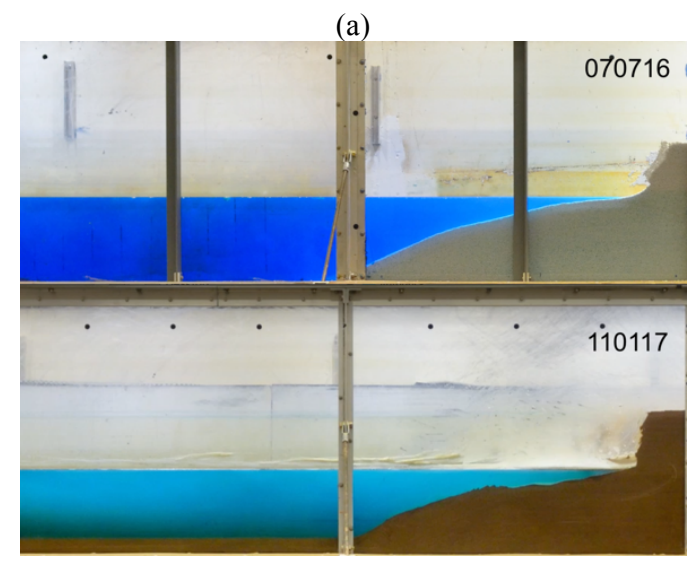

(b)

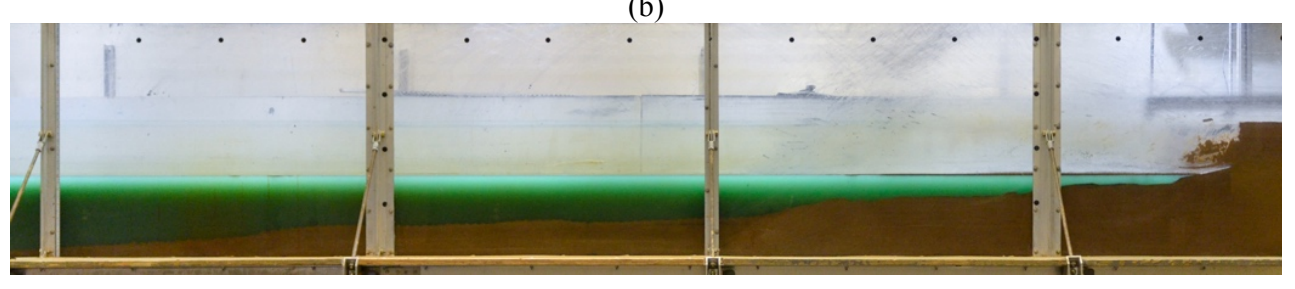

Fig. 5. (a) 30 embankment profiles 30 minutes of wave action ( 3 active interval); (b) 33 hours of wave action.

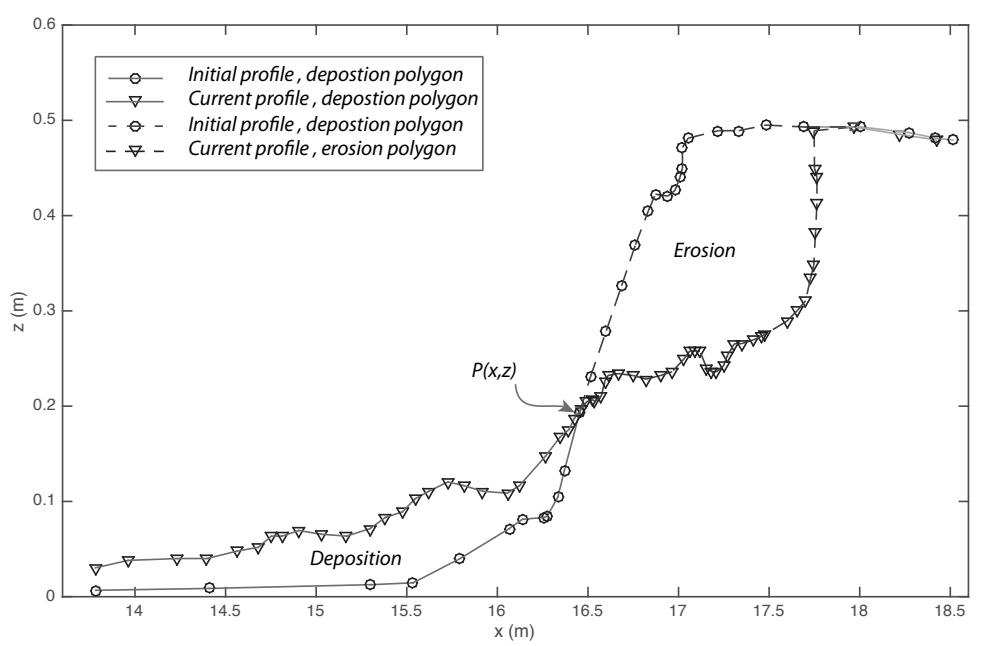

Fig. 6. Estimation of erosion and deposition areas. 
In Figure 7, erosion area and retreat time series are compared. Retreat is defined as the location of the top of the scarp relative to the offshore end of the embankment top. Power functions were fitted to the linear segment (log-log scale) of the data in Fig 7. Erosion and retreat rates were initially high and nonlinear. Once the scarp face developed and wave breaking started, erosion and retreat slowed down with a constant exponent, or almost stopped in the case of the non-cohesive embankment (110117). Although both models advanced to the same profile after 30 minutes, the erosion rate of the cohesive embankment was much higher than the non-cohesive embankment during later stages. For the experiments, finer particles in the cohesive embankment were suspended, allowing them to be transported further offshore; therefore, more material was eroded from the toe of the scarp to balance losses in the breaking zone.

(a)

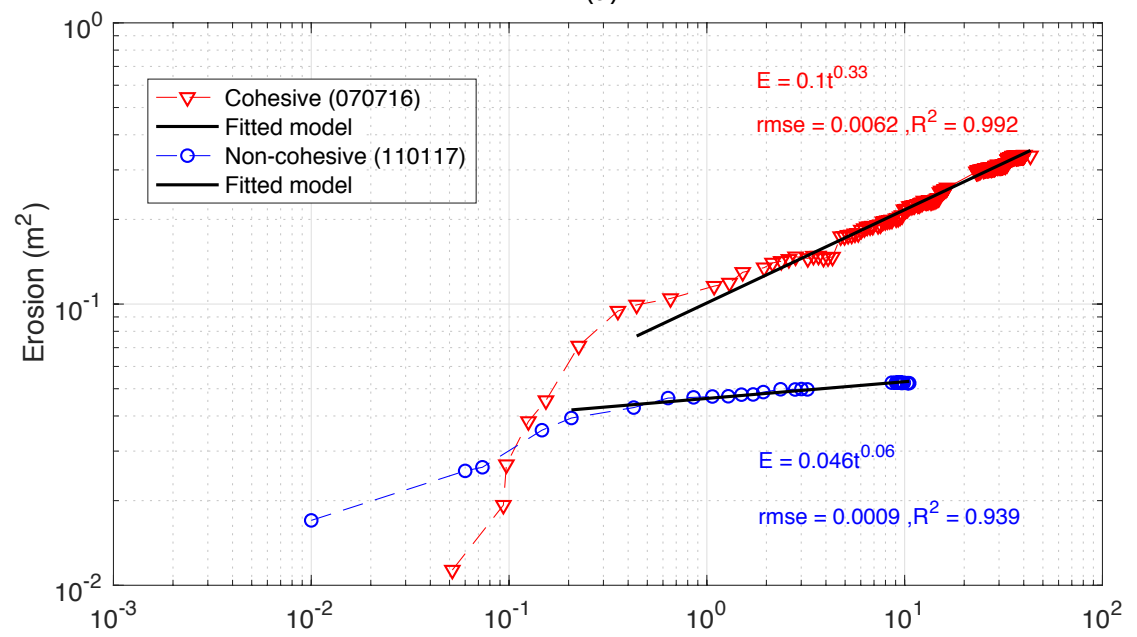

(b)

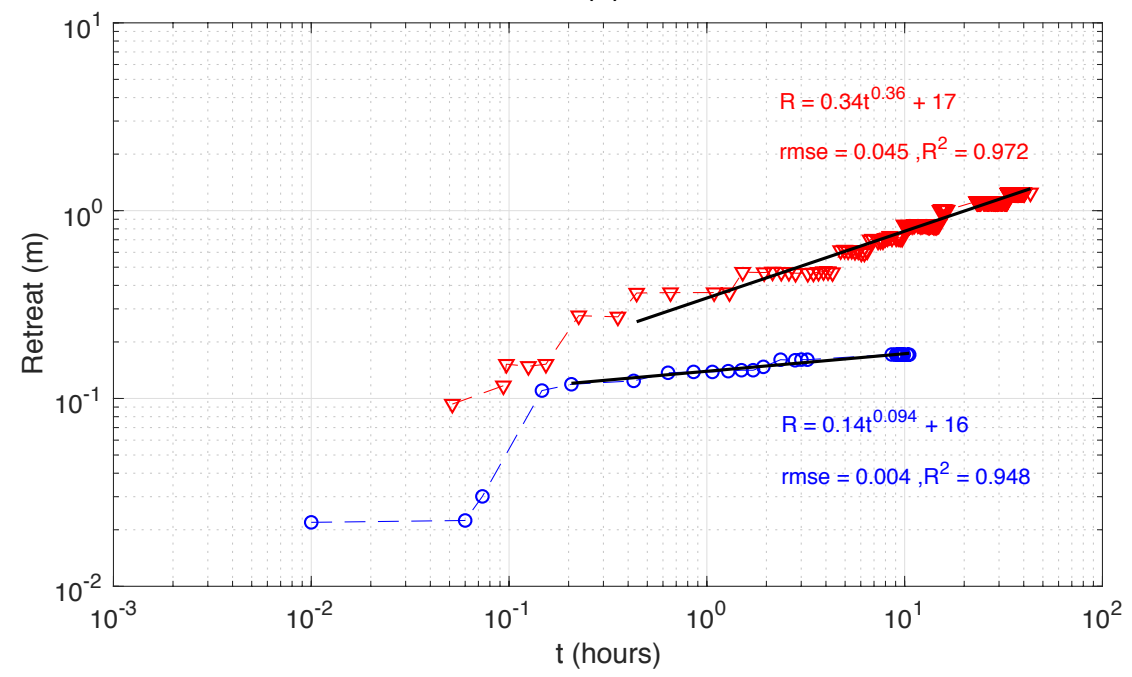

Fig. 7. Time history of erosion (a) and retreat (b) of cohesive and non-cohesive embankment. 


\section{Conclusions}

Laboratory experiments were carried out to investigate wave erosion and retreat of embankments constructed from cohesive and non-cohesive materials. The results of the experiments showed that the cohesive embankment, composed mostly of silt, initially eroded at a similar rate relative to the non-cohesive embankment composed of artificial particles. While an equilibrium beach profile was established very quickly for the noncohesive material, the cohesive embankment profile continued eroding for a much longer period. For both cases, the erosion process was in discrete slump failures, and both erosion and retreat declined exponentially with a constant exponent.

Glenn Gray and Will Andrews provided invaluable support during preparation of the experiments.

\section{References}

1. Wren, D. G., Ozeren, Y., Taylor, J., and Reba, M. L. Assessment of irrigation reservoir levee impairment in Arkansas, USA. Journal of Soil and Water Conservation. (Accepted 1/8/2018)

2. Wren, D. G., Ozeren, Y., and Reba, M. L. Measuring the erosion of an irrigation reservoir levee. Transactions of the American Society of Agricultural and Biological Engineers. Transactions of the ASABE. 59, 1 pp. 41-48 (2016)

3. van Rijn, L. C. Coastal erosion and control. Ocean Coastal Mgmt., 54,12 pp. 867-887 (2011)

4. Bishop, C., Skafel, M., \& Nairn, R. Cohesive profile erosion by waves. In Coastal Engineering Conference 3, pp. 2976-2976 ASCE (1992)

5. Skafel, M. G. Laboratory measurement of nearshore velocities and erosion of cohesive sediment (till) shorelines. Coastal Engineering 24, 3-4 pp. 343-349 (1995) 